\title{
Dynamics of perturbations in disordered chaotic systems
}

\author{
Ivan G. Szendro, ${ }^{1,2, *}$ Juan M. López, ${ }^{1, \dagger}$ and Miguel A. Rodríguez ${ }^{1, \$}$ \\ ${ }^{1}$ Instituto de Física de Cantabria (IFCA), CSIC-UC, E-39005 Santander, Spain \\ ${ }^{2}$ Departamento de Física Moderna, Universidad de Cantabria, Avenida Los Castros, E-39005 Santander, Spain
}

(Received 27 February 2008; published 4 September 2008)

\begin{abstract}
We study the time evolution of perturbations in spatially extended chaotic systems in the presence of quenched disorder. We find that initially random perturbations tend to exponentially localize in space around static pinning centers that are selected by the particular configuration of disorder. The spatiotemporal behavior of typical perturbations $\delta u(x, t)$ is analyzed in terms of the Hopf-Cole transform $h(x, t) \equiv \ln |\delta u(x, t)|$. Our analysis shows that the associated surface $h(x, t)$ self-organizes into a faceted structure with scale-invariant correlations. Scaling analysis of critical roughening exponents reveals that there are three different universality classes for error propagation in disordered chaotic systems that correspond to different symmetries of the underlying disorder. Our conclusions are based on numerical simulations of disordered lattices of coupled chaotic elements and equations for diffusion in random potentials. We propose a phenomenological stochastic field theory that gives some insights on the path for a generalization of these results for a broad class of disordered extended systems exhibiting space-time chaos.
\end{abstract}

DOI: 10.1103/PhysRevE.78.036202

PACS number(s): 05.45.Jn, 05.45.Ra, 05.40.-a

\section{INTRODUCTION}

Spatially extended chaotic systems (SECS) are of great importance for the understanding of fundamental problems in deterministic many-particle systems, including hydrodynamics and turbulence [1,2] or weather forecasting [3]. These systems exhibit dynamical instabilities that can be quantified by the spectrum of Lyapunov exponents and their corresponding Lyapunov vectors (LVs) [1,4-6].

Recently, the application of some tools and concepts borrowed from the physics of nonequilibrium statistical systems has been shown to be a very promising line of research to study certain aspects of SECS. In particular, it has been observed that (after a suitable logarithmic transformation) the evolution of an infinitesimal perturbation in deterministic SECS can be generically described as a scale-invariant rough surface [7-11]. In this surface picture, the erratic fluctuations of the system, due to the chaotic dynamics, are interpreted as noise. Remarkably, it has been shown $[7,8,11]$ that in many cases the associated surfaces belong to the Kardar-ParisiZhang (KPZ) [12] universality class of nonequilibrium surface roughening. Nonetheless, the probability distribution of the randomness generated by the chaotic trajectory can be crucial. In this regard, the existence of long-range correlations [9] or a fat tail of the distribution [10] may change the universality class observed in the surface growth picture.

Typically the above discussed studies deal with homogeneous systems, where only identical elements are coupled, often in a diffusive manner. However, much less is known about the evolution of chaotic perturbations in inhomogeneous extended systems, where the many coupled elements

\footnotetext{
*szendro@ifca.unican.es; Present address: Max Planck Institute for the Physics of Complex Systems, Nöthnitzer Strasse 38, 01187 Dresden, Germany

†lopez@ifca.unican.es

†rodrigma@ifca.unican.es
}

are either different or the coupling itself is a quenched random field that varies along the system. Such conditions naturally arise in some important applications, e.g., in regional weather forecasting, where inhomogeneity is present in the form of explicit surface-topography dependent terms $[3,13-15]$ in the dynamic equations. Inhomogeneous SECS can indeed demonstrate rather unusual properties, such as, for instance, taming of spatiotemporal chaos induced by disorder [16], disorder-enhanced synchronization [17,18], and avoided crossing and level repulsion similar to that occurring for energy eigenvalues in disordered quantum systems [19].

In this paper, we focus on the dynamics of perturbations in disordered SECS, which is not covered much in the literature. We analyze here a rather simple but enlightening model consisting of a lattice of coupled chaotic elements whose parameters are randomly distributed or the coupling among them is a quenched random variable. We show that longrange temporal correlations, induced by the quenched disorder, can cause perturbations to strongly localize. The strong localization observed here is essentially different from the dynamical localization that has been previously reported for homogeneous SECS $[7,8]$. In the latter, the positions of the localization centers keep fluctuating in space, while in the presence of quenched disorder we find that the positions of the localization centers are fixed for a given disorder realization. We analyze typical perturbations $\delta u(x, t)$ by making use of the Hopf-Cole transform $h(x, t) \equiv \ln |\delta u(x, t)|$ and mapping to the equivalent surface growth problem. Strong localization leads to the formation of faceted structures in the corresponding surface picture. These Lyapunov surfaces also exhibit a coarsening behavior and anomalous kinetic roughening, akin to some nonequilibrium growing surfaces. The connection of the problem of the propagation of errors in disordered SECS with the problem of diffusion in random potentials is also discussed. We propose a phenomenological stochastic field theory pointing toward a generalization of our results for a broad class of disordered extended systems exhibiting space-time chaos. 


\section{DESCRIPTION OF THE MODELS}

We focus our numerical study on coupled-map lattices with quenched disorder. We consider $L$ coupled chaotic maps $u_{x}(t)$, with $x=1,2, \ldots, L$, following the evolution equation

$$
\begin{aligned}
u_{x}(t+1)= & \sigma_{x, x+1} f_{x+1}\left[u_{x+1}(t)\right]+\sigma_{x, x-1} f_{x-1}\left[u_{x-1}(t)\right] \\
& +\left(1-2 \sigma_{x, x}\right) f_{x}\left[u_{x}(t)\right]
\end{aligned}
$$

where the $\sigma_{x, x \pm 1}$ are the disordered nearest-neighbor coupling constants, $\sigma_{x, x}$ is the on-site contribution, and $f_{x}$ is the local nonlinear map at site $x$. Here we choose $f_{x}(\varrho)$ $=c_{x}(1 / 2-|\varrho \bmod 1-1 / 2|)$, which is a periodic continuation of the tent map. This map shows chaotic behavior for $c_{x}$ $\in(1, \infty)$, with a Lyapunov exponent that grows logarithmically with $c_{x}$. This choice allows one to study extended coupled systems with a spatial distribution of Lyapunov exponents by allowing $c_{x}$ to take randomly distributed values along the lattice.

Almost any initial random perturbation $\delta u_{x}(t=0)$ will grow in magnitude and develop space-time correlations while propagating along the system, quickly aligning with the most unstable direction in tangent space, the so-called main LV:

$$
\begin{aligned}
\delta u_{x}(t+1)= & \sigma_{x, x+1} f_{x+1}^{\prime}\left[u_{x+1}(t)\right] \delta u_{x+1}(t) \\
& +\sigma_{x, x-1} f_{x-1}^{\prime}\left[u_{x-1}(t)\right] \delta u_{x-1}(t) \\
& +\left(1-2 \sigma_{x, x}\right) f_{x}^{\prime}\left[u_{x}(t)\right] \delta u_{x}(t),
\end{aligned}
$$

where $f_{x}^{\prime}(\varrho)$ is just the derivative of the local map $f_{x}(\varrho)$ with respect to its argument $\varrho$.

Numerical integration of the tangent space equations (1) looks apparently rather simple, however, a caveat is in order. We found that the introduction of quenched disorder leads to the appearance of very large differences among error field $\delta u_{x}(t)$ values at certain sites of the system. The reason for this is the quenched nature of the disorder so that, if a large value of the random variable is assigned to a given site, it will continue giving high contributions for all times. In fact, these differences can become so large during the simulation that, if we were to naively integrate Eq. (1) and just multiply by some global factor to avoid numerical overflow, the perturbation field at those sites where the perturbation values are small would soon be considered as zero by the computer due to accuracy limitations. It is rather simple to overcome this technical problem by avoiding calculating the $\delta u_{x}(t)$ directly, but rather computing the quotients $\vartheta_{x}(t+1)=\delta u_{x}(t$ $+1) / \delta u_{x}(t)$ and $\varphi_{x}(t)=\delta u_{x+1}(t) / \delta u_{x}(t)$ instead. We therefore rewrite Eq. (1) as

$$
\begin{gathered}
\vartheta_{x}(t+1)=\sigma_{x, x+1} f_{x+1}^{\prime}\left[u_{x+1}(t)\right] \varphi_{x}(t) \\
+\sigma_{x, x-1} f_{x-1}^{\prime}\left[u_{x-1}(t)\right] / \varphi_{x-1}(t)+\left(1-2 \sigma_{x, x}\right) f_{x}^{\prime}\left[u_{x}(t)\right], \\
\varphi_{x}(t+1)=\varphi_{x}(t) \frac{\vartheta_{x+1}(t+1)}{\vartheta_{x}(t+1)} .
\end{gathered}
$$

Now we can numerically integrate this pair of equations instead of Eq. (1). This useful numerical trick resolves the problem of possible overflows during the simulations.

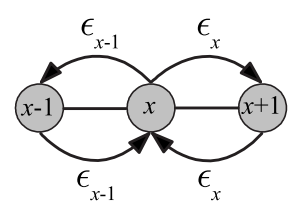

(a)

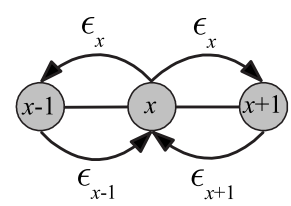

(b)
FIG. 1. The coupling configurations of one site to its neighboring sites and vice versa for (a) the random barrier (RB) and (b) random trap (RT) model.

We have studied three different scenarios for a disordered couple-map lattice system. In the first scenario (model A) we consider the case in which the couplings are identical along the system, but coupled elements are inhomogeneous. In the simplest setting we can model this situation by drawing the local map constant $c_{x}$ from a uniform distribution, so we have

$$
\text { model A: }\left\{\begin{array}{l}
c_{x} \in \mathrm{U}(1,2), \\
\sigma_{x, y}=1 / 3,
\end{array}\right.
$$

where $y \in\{x-1, x, x+1\}$. Note that we chose a homogeneous democratic coupling $\left(\sigma_{x, y}=1 / 3\right)$ all along the system. In this situation individual elements are more chaotic or less chaotic depending on the corresponding value of $c_{x}$. Larger values of $c_{x}$ lead to a locally faster growth of the perturbation at site $x$. Strictly speaking, this is only true for uncoupled maps. Things are much more involved here since the maps are coupled. Thus, the behavior of the perturbation at one site does not only depend on the map at that specific site, but also on the dynamics of its neighborhood.

A different source of disorder we have explored is the existence of a quenched random coupling, while the individual maps are all identical. This corresponds to systems with a disordered diffusion coefficient. From the classical theory of diffusion in disordered media we know that two different symmetries are of interest [20,21]. These two models are sketched in Fig. 1 and described in detail below.

On the one hand, we have the random barrier (RB) model [20,21]. Physically this corresponds to the existence of a random potential barrier between every two neighboring sites so that the diffusion coefficient from site $x$ to site $x+1$ is equal to that from site $x+1$ to site $x$ (see Fig. 1). In other words, the disorder is associated with the bonds connecting neighboring sites on the lattice. We call this configuration model B:

$$
\text { model B: }\left\{\begin{array}{l}
c_{x}=2, \\
\sigma_{x, x-1}=\epsilon_{x}, \\
\sigma_{x, x+1}=\epsilon_{x+1}, \\
\sigma_{x, x}=\left(\epsilon_{x}+\epsilon_{x+1}\right) / 2,
\end{array}\right.
$$

where the disorder is drawn from a uniform distribution $\epsilon_{x}$ $\in \mathrm{U}\left(0, \epsilon_{0}\right)$ and $\epsilon_{0}$ being an arbitrary parameter that we take as $1 / 3$ unless otherwise stated.

On the other hand, some disordered systems are better described by the so-called random trap (RT) model [20,21]. In this case a static disordered diffusion coefficient is as- 


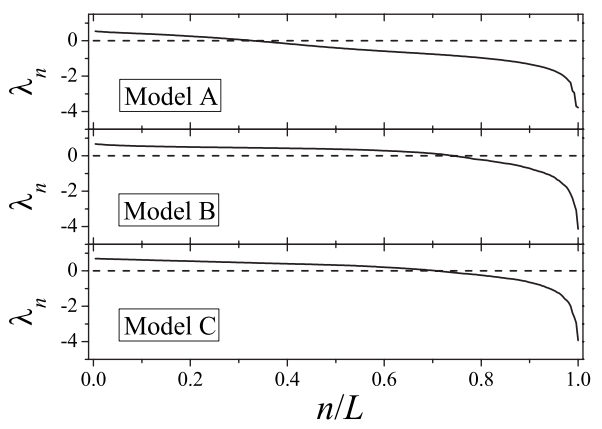

FIG. 2. Lyapunov spectra for models A, B, and C in a lattice of $L=256$ coupled tent maps after average over ten different disorder realizations.

signed to each lattice site $x$ instead to bonds. This corresponds to our model $\mathrm{C}$, which is defined by

$$
\text { model C: }\left\{\begin{array}{l}
c_{x}=2, \\
\sigma_{x, x-1}=\epsilon_{x-1}, \\
\sigma_{x, x+1}=\epsilon_{x+1}, \\
\sigma_{x, x}=\epsilon_{x},
\end{array}\right.
$$

where the disorder is uniformly distributed, $\epsilon_{x} \in \mathrm{U}\left(0, \epsilon_{0}\right)$, and again $\epsilon_{0}$ can be any number in the interval $(0,1 / 2)$, here we use $\epsilon_{0}=1 / 3$.

Typical Lyapunov spectra corresponding to the three disordered models introduced here are shown in Fig. 2 for a system of size $L=256$ and averages over 10 different disorder realizations. One can see that, depending on the model, one has $30-80 \%$ of the spectra in the region $\lambda>0$. Note that Lyapunov spectra are self-averaging, i.e., as we increase the system size, Lyapunov spectra for a specific disorder realization approach the spectrum averaged over disorder realizations.

Some remarks are now in order. First, let us stress that these two disordered diffusion configurations, namely, RB and RT, were not chosen arbitrarily, but are in fact all existing physically meaningful ways to introduce disorder in the diffusive couplings [20,21]. Second, it is important to remark that in this paper we focus on systems in the presence of weak disorder, where the probability density of having zero disorder at any given site $\mathcal{P}(\epsilon=0)$ is bounded. We do leave out of our study the case of strong disorder $\mathcal{P}(\epsilon \rightarrow 0) \sim \epsilon^{-|\nu|}$, where a fraction of the system sites can effectively act as sinks, which is known to have a great impact on the asymptotic transport properties $[20,21]$ and will be discussed elsewhere.

In principle, classical results on the problem of diffusion in random media $[20,21]$ may also be invoked to argue that the details of the disorder distribution should be irrelevant in the sense that weak disorder gives rise to the same asymptotic dynamical behavior independently of the detailed form of the disorder distribution. The same conclusion applies in the case of strong disorder, where the asymptotic dynamics is fully determined by the form of the divergence at the origin, i.e., the value of the exponent $\nu$. In the following we focus on the case of weak disorder and a uniform distribution $\mathcal{P}(\epsilon)=\mathrm{U}\left(0, \epsilon_{0}\right)$ with the parameter $\epsilon_{0}=1 / 3$ is

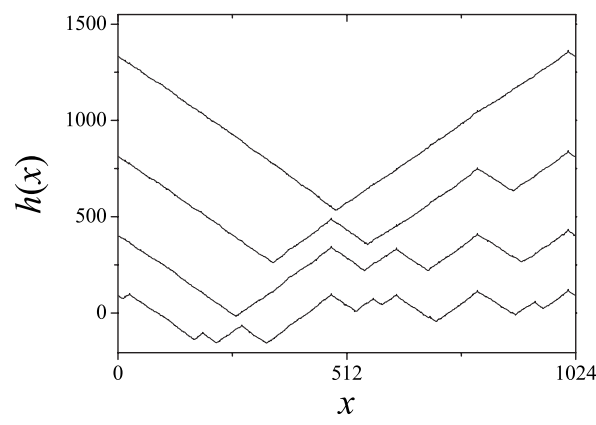

FIG. 3. Typical evolution of a Lyapunov vector surface $h(x, t)$. As time evolves (from bottom to top) one can observe the emergence of facets that grow until, in the long time limit, the whole interface is spanned by one single facet. Tiny fluctuations can be observed at small scales. The data correspond to model $\mathrm{C}$ in a lattice of $L=1024$ maps.

used to exemplify our results. However, in the presence of random multiplicative terms the critical behavior might be affected by the detailed form of the disorder distribution [22].

\section{SURFACE EVOLUTION AND COARSENING}

As occurs in the case of homogeneous systems, the dynamics of the main LV can be conveniently described in terms of the equivalent surface picture [7-11]. The reason is that the logarithm of the perturbation field turns out to be scale invariant, so that correlations have the form of powerlaw functions and critical exponents can then be used to characterize the space-time structure of perturbations. Therefore, in this paper we are interested in the scaling properties of the rough surface defined by the Hopf-Cole transform of the perturbation $h(x, t) \equiv \ln \left|\delta u_{x}(t)\right|=\ln \left|\delta u_{x}(0)\right|$ $+\sum_{\tau=1}^{t} \ln \left|\vartheta_{x}(\tau)\right|$.

Space-time scaling properties of chaotic perturbations in inhomogeneous systems turn out to be very different from those in homogeneous systems, even in the case of weak disorder studied here. As can be immediately seen in Fig. 3, a first observation is the patterned structure of the LV surface, which is visible to the naked eye and contrasts with the KPZ-type morphologies observed in homogeneous systems $[7,11]$. This already indicates that significant differences are to be expected in the scaling properties of inhomogeneous systems. Even weak disorder induces self-organization in a triangular structure, which reflects the strong spatial localization of the perturbation in the form of a exponential profile around some strong pinning centers. Note that a facet $h(x, t)=h\left(x_{0}\right)-s(t)\left|x-x_{0}\right|$ with a cusp at $x=x_{0}$ and slope $s(t)$ corresponds to an exponential profile of the perturbation $\delta u_{x}(t) \propto \exp \left[-s(t)\left|x-x_{0}\right|\right]$ around the pinning center $x_{0}$.

In Fig. 3 we plot a typical evolution of the main LV according to Eq. (1) in a system of size $L=1024$ for a given disorder realization in the case of model $\mathrm{C}$. We observe that at short times, the surface $h(x, t)$ is composed of triangular facets of varying sizes which grow in time until, in the long time limit, the whole interface is formed by just one single facet. Therefore, as time evolves the size of the triangles 


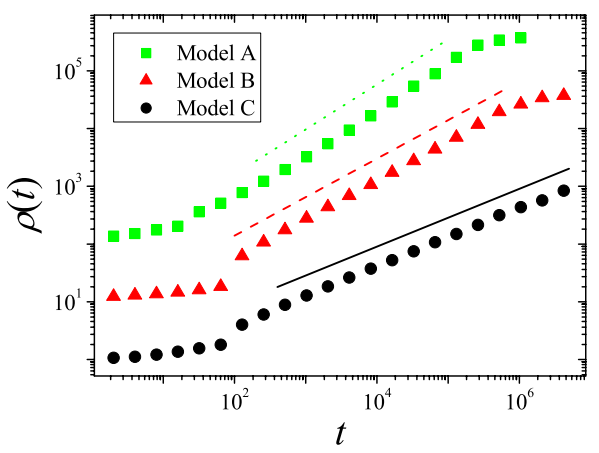

FIG. 4. (Color online) The evolution of the lateral mound size $\rho(t)$ is plotted for the three models. The straight lines are plotted to guide the eye and have slopes 0.78 (dotted), 2/3 (dashed), and 1/2 (solid), respectively. The curves for models A and $\mathrm{B}$ have been shifted for better visibility.

increases and number of triangles diminishes. This surface dynamics corresponds to nonequilibrium "coarsening." Similar triangular morphologies are obtained for the three models $\mathrm{A}, \mathrm{B}$, and $\mathrm{C}$ introduced above. Close inspection and analysis of critical exponents shows that the three models actually belong to different universality classes.

In order to characterize the growth of the faceted pattern we have measured the lateral mound size $\rho(t)$, which gives the coarsening length or typical length scale of the instability. This is usually done by calculating the slope-slope correlation function $\langle\overline{\nabla h(x, t) \nabla h(x+r, t)}\rangle$ and measuring the distance, $\rho(t)$, at which this correlation crosses zero. We calculate the surface gradient as the centered discrete derivative $\nabla h(x, t) \equiv[h(x+1, t)-h(x-1, t)] / 2$. In Fig. 4 the lateral mound size, $\rho(t)$, for the three models is shown. After a short initial period, $\rho(t)$ grows as a power law in time with exponents $\theta_{\mathrm{B}}=0.67 \pm 0.03$ and $\theta_{\mathrm{C}}=0.48 \pm 0.03$, for models $\mathrm{B}$ and $\mathrm{C}$, respectively. At long times $\rho(t)$ saturates, as the surface becomes dominated by a single facet and the coarsening length becomes comparable to the system size. For model A the intermediate region does not seems to be following a simple power law. Nonetheless, we have fitted an exponent $\theta_{\mathrm{A}}=0.78 \pm 0.05$ for the sake of comparison. The different scaling exponents for the coarsening length already indicate that the three models possibly belong to different universality classes.

\section{SURFACE ROUGHNESS SCALING}

We will now perform a scaling analysis of the Hopf-Cole transform. The idea behind this analysis is to obtain information about the spatiotemporal correlations in order to identify the possible universality classes corresponding to different symmetries, which might help to identify physical mechanisms underlying real data.

Kinetic roughening of random scale-invariant surfaces is usually described by the so-called Family-Vicsek scaling ansatz $[23,24]$. However, faceted surfaces can often exhibit anomalous scaling properties in the sense that local and global fluctuations may scale with different scaling functions (and exponents). The existence of anomalous scaling, as op- posed to standard Family-Vicsek scaling, was first reported in the context of surface growth models by Amar et al. [25], and the need to introduce more general scaling ansatzes was soon after noticed by Schroeder et al. [26] as well as by Das Sarma et al. [27] in the case of the so-called super-rough anomalous scaling. These results were later shown to be just particular cases of anomalously scaling correlations that can be understood as subclasses of a generic scaling theory of surface growth [28]. As it turns out, the present faceted scaleinvariant surfaces do exhibit anomalous scaling properties similar to those reported in Ref. [29], which are captured by the generic dynamic scaling form introduced by Ramasco et al. [28]. In this section we briefly describe the generic scaling theory for surface kinetic roughening that will be used later on to analyze our numerical data.

The roughness of scale-invariant surfaces corresponds to the fluctuations of the surface height. These fluctuations can be computed either locally or globally as follows. On the one hand, the global roughness exponent $\alpha$ can be obtained from the scaling behavior of the global width $W(L, t)$ $=\left\langle\overline{[h(x, t)-\bar{h}(t)]^{2}}\right\rangle^{1 / 2}$. Here, the overline denotes an average over all sites $x$ in a system of size $L$ and brackets denote the average over different realizations. For scale-invariant surfaces one expects the global width to scale as $W(L, t)$ $=t^{\alpha / z} \mathcal{G}\left(L / t^{1 / z}\right)$, where $\mathcal{G}(u)$ is a scaling function that becomes constant for $u \gg 1$ and decays as $\sim u^{\alpha}$ for $u \ll 1$. The roughness exponent $\alpha$ and dynamic exponent $z$ characterize the scaling behavior of the global surface fluctuations.

On the other hand, one can measure the local roughness exponent $\alpha_{\text {loc }}$, which is defined via the scaling behavior of the local width $w(l, t)=\left\langle\left\langle\left[h(x, t)-\langle h\rangle_{l}(t)\right]^{2}\right\rangle_{l}\right\rangle^{1 / 2}$, where $\langle\cdots\rangle_{l}$ denotes an average over $x$ in a window of size $l$. The local width scales as $w(l, t) \sim t^{\alpha / z} \mathcal{G}_{\mathrm{A}}\left(l / t^{1 / z}\right)$, where the scaling function $\mathcal{G}_{\mathrm{A}}(u)$ has a similar asymptotic behavior as $\mathcal{G}(u)$, but with a local anomalous exponent, $\sim u^{\alpha_{\text {loc }}}$ for $u \ll 1$. So that in the stationary regime (for $t \gg L^{z}$ ) one obtains $w_{\text {stat }}(l, L)$ $\sim l^{\alpha_{\mathrm{loc}}} L^{\alpha-\alpha_{\mathrm{loc}}}$. In the cases where local and global roughness exponents do not coincide the scaling is said to be anomalous.

Following Ramasco et al. [28], in order to correctly classify the different forms that scaling can take it is convenient to introduce a third roughness exponent, namely the so called spectral roughness exponent $\alpha_{\mathrm{s}}$. This is defined in terms of the structure factor (or power spectrum in $k$ space)

$$
S(k, t)=\langle\hat{h}(k, t) \hat{h}(-k, t)\rangle
$$

where $\hat{h}(k, t)$ is the spatial Fourier transform of $h(x, t)-\bar{h}(t)$. Ramasco et al. [28] showed that scale-invariant roughening in $d+1$ dimensions is fully described in the following scaling ansatz:

$$
S(k, t)=k^{-(2 \alpha+d)} s\left(k t^{1 / z}\right),
$$

$z$ being the dynamical exponent that connects temporal scales $\tau$ and length scales $l$ according to $\tau \sim l^{z}$, and the nontrivial scaling function takes the form 


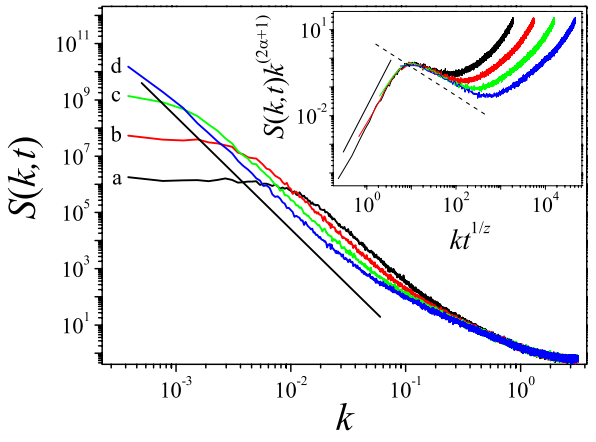

FIG. 5. (Color online) The spectral power functions of the surfaces obtained for model A are plotted for times $t=2^{12}(\mathrm{a}), 2^{14}(\mathrm{~b})$, $2^{16}(\mathrm{c}), 2^{18}(\mathrm{~d})$. The straight line corresponds to $\alpha_{s}=1.5$. In the inset we show the data collapse according to the scaling ansatz (6) and (7), using $\alpha=1.03$ and $z=1.3$. The dashed and the solid straight line have slopes -1 and 3 , respectively. The plotted data correspond to model A for a system of size $L=16384$ averaged over 100 disorder realizations.

$$
s(u) \sim\left\{\begin{array}{cl}
u^{2\left(\alpha-\alpha_{s}\right)} & \text { if } u \gg 1, \\
u^{2 \alpha+d} & \text { if } u \ll 1 .
\end{array}\right.
$$

Here $\alpha$ is the global roughness exponent. With the help of these three exponents one can distinguish the four different possible types of scaling that any scale-invariant rough surface can exhibit,

$$
\begin{gathered}
\text { if } \alpha_{\mathrm{s}}<1 \Rightarrow \alpha_{\mathrm{loc}}=\alpha_{\mathrm{s}} \begin{cases}\alpha_{\mathrm{s}}=\alpha \Rightarrow & \text { Family-Vicsek, } \\
\alpha_{\mathrm{s}} \neq \alpha \Rightarrow & \text { intrinsic }\end{cases} \\
\text { if } \alpha_{\mathrm{s}}>1 \Rightarrow \alpha_{\mathrm{loc}}=1 \begin{cases}\alpha_{\mathrm{s}}=\alpha \Rightarrow & \text { super-rough } \\
\alpha_{\mathrm{s}} \neq \alpha \Rightarrow & \text { faceted }\end{cases}
\end{gathered}
$$

Note that only in the case where standard Family-Vicsek scaling is valid, which means $\alpha=\alpha_{\mathrm{loc}}=\alpha_{\mathrm{s}}$, the surface is selfaffine. In the other cases this will in general not be true, i.e., local and global fluctuations will still exhibit dynamical scaling, but will do so with different exponents.

We now use this general scaling ansatz to study the scaling properties of our system. In order to do so, we have calculated the structure factor corresponding to the HopfCole transforms of the perturbations at various times. Figures 5-7 show the structure factor for models A, B, and C, respectively. We observe a power-law decay as $\sim k^{-\left(2 \alpha_{s}+1\right)}$ for momenta limited by a large scale and a small scale cutoff. At large length scales the power-law behavior is limited by the correlation length $\xi(t) \sim t^{1 / z}$, as expected from dynamical scaling. The cutoff at short lengths also grows in time, suggesting the existence of a different dynamics at small scales, which will be studied in detail in Sec. V. Note that the curves $S(k, t)$ shift downward for increasing times, indicating the presence of anomalous scaling with $\alpha_{\mathrm{s}}>\alpha$. Specifically, one expects $S(k, t) \sim k^{2 \alpha_{\mathrm{s}}+1} t^{\left(\alpha-\alpha_{\mathrm{loc}} / z\right.}$. We have measured $\alpha_{\mathrm{s}}$ $=1.43 \pm 0.05,1.47 \pm 0.05$, and $1.45 \pm 0.05$ for models A, B, and $\mathrm{C}$, respectively. This should be compared with the value of $\alpha_{\mathrm{s}}=3 / 2$ that can be obtained analytically for interfaces consisting of random smooth facets [28]. In the insets of Figs. 5-7 we show a data collapse according to the generic

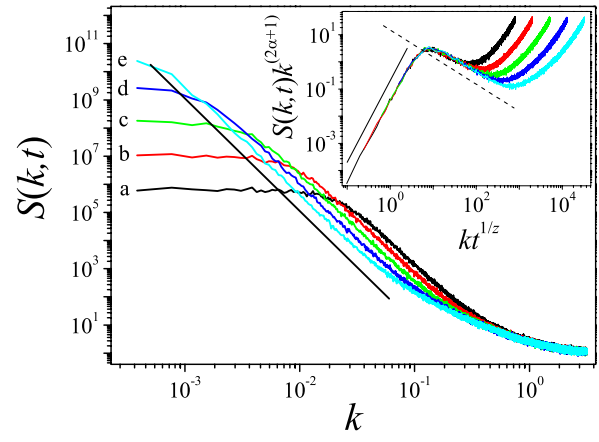

FIG. 6. (Color online) The spectral power functions of interfaces obtained for model B are plotted for times $t=2^{12}(\mathrm{a}), 2^{14}(\mathrm{~b}), 2^{16}(\mathrm{c})$, $2^{18}(\mathrm{~d}), 2^{20}(\mathrm{e})$. The straight line corresponds to $\alpha_{s}=1.5$. In the inset we show the data collapse according to scaling ansatz (6) and (7), using $\alpha=1.0$ and $z=1.5$. The dashed and the solid straight line have slopes -1 and 3 , respectively. The plotted data corresponds to model B for a system of size $L=16384$ averaged over 100 realizations.

scaling ansatz in Eqs. (6) and (7), obtaining values for the roughness and dynamical exponents $\alpha$ and $z$. As can be verified, all three models show scaling properties well described by the faceted interface scaling class in Ramasco's classification, Eq. (8), with a roughness exponent $\alpha \approx 1$ for all the three models. Nonetheless, all three systems yield different values for the dynamical exponent, $z_{\mathrm{A}}=1.3 \pm 0.1, \quad z_{\mathrm{B}}$ $=1.50 \pm 0.03$, and $z_{\mathrm{C}}=2.00 \pm 0.03$, again indicating that the three models belong to different universality classes. These values of $z$ define the typical length scale that correlations have spread along the system up to time $t, \xi(t) \sim t^{1 / z}$, and are in good agreement with the inverse of the exponents $(\theta$ $=1 / z$ ) that we obtained from the analysis of the coarsening length discussed in Sec. III for all the three models studied in this paper.

\section{SEPARATING FACETS FROM FLUCTUATIONS}

The scaling analysis of the surface fluctuations presented in the preceding sections has shown that the LV surface self-

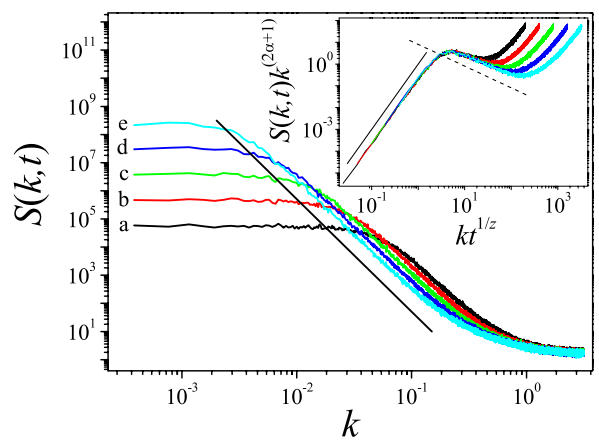

FIG. 7. (Color online) The spectral power functions of interfaces obtained for model $\mathrm{C}$ are plotted for times $t=2^{12}(\mathrm{a}), 2^{14}(\mathrm{~b}), 2^{16}(\mathrm{c})$, $2^{18}(\mathrm{~d}), 2^{20}(\mathrm{e})$. The straight line corresponds to $\alpha_{s}=1.5$. In the inset we show the data collapse according to scaling ansatz (6) and (7), using $\alpha=1.0$ and $z=2.0$. The dashed and the solid straight line have slopes -1 and 3 , respectively. The plotted data corresponds to model C for a system of size $L=16384$ averaged over 100 realizations. 


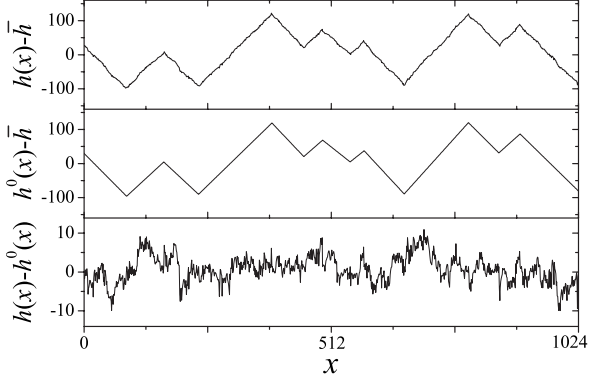

FIG. 8. For a segment of 1024 maps on a system of $L=16364$ we exemplify the separation of the surface $h(x)$ into the triangular pattern $h^{0}(x)$ and the difference $y(x)=h(x)-h^{0}(x)$, which gives the small scale fluctuations.

organizes in a characteristic triangular pattern for all the three models of disorder. A closer inspection, in particular the computation of the structure factor, has also revealed the existence of a cutoff at small scales such that length scales below that point obey a different dynamics. This suggests the existence of another dynamical process taking place at small scales which is different from the mechanism responsible for the large scale faceted structure.

In this section we show that one can actually separate two different contributions to the LV surface height: The large scale pattern $h^{0}(x, t)$ formed by facets of constant slope and a randomly fluctuating term $y(x, t)$ which becomes the dominant one at small scales. Therefore the surface profile at any given time can be expressed as the sum of the two independent components as $h(x, t)=h^{0}(x, t)+y(x, t)$. This separation can be carried out numerically as follows. For a given time the interface $h(x, t)$ is smoothed to remove local maxima and minima corresponding to local fluctuations upon the triangular structure. This is done by replacing the heights at every site by a spatial average over some arbitrary region around each site. Then, the locations of the cusp sites and valley sites of the smoothed curve are easily identified and the faceted pattern $h^{0}(x)$ is defined as the set of straight lines connecting the cusp points to the neighboring valley points. The interface corresponding to the fluctuations is then obtained by taking the difference $y(x, t)=h(x, t)-h^{0}(x, t)$ as exemplified in Fig. 8.

Interestingly, one can observe that the triangular pattern $h^{0}$ and the random fluctuation components $y(x, t)$ are uncorrelated. This can be shown by checking that $\left\langle\hat{h}^{0}(k, t) \hat{y}(-k, t)\right\rangle=0$, which is equivalent to proving the identity

$$
S(k, t)=\left\langle\widehat{h^{0}}(k, t) \widehat{h^{0}}(-k, t)\right\rangle+\langle\hat{y}(k, t) \hat{y}(-k, t)\rangle .
$$

In fact, the numerical data presented in Fig. 9 confirm that both components are actually uncorrelated. Figure 9 shows the structure factor of the faceted pattern $h^{0}(x, t)$, the local fluctuations $y(x, t)$, and the complete interface $h(x, t)$ at two different times. In particular, the data shown correspond to model $\mathrm{B}$, but we obtained identical results for for the other two models.

It becomes apparent that the anomalous scaling of the surface stems from that of the faceted structure. Since the

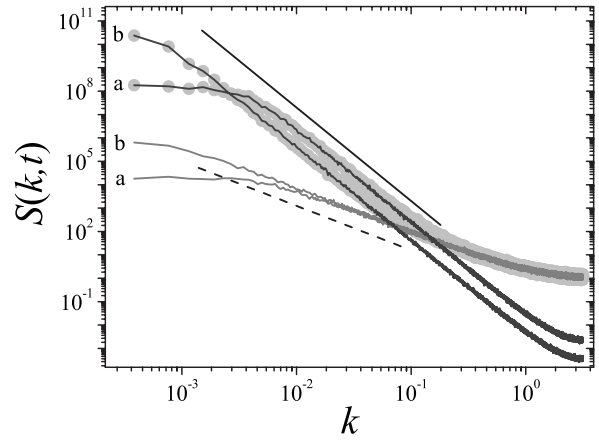

FIG. 9. The power spectra of the interface $h(x)$ (light gray dots), the triangular structure $h^{0}(x)$ (dark gray lines), and the difference $y(x)=h(x)-h^{0}(x)$ (gray lines) are plotted for two different times $t_{a}$ $<t_{b}$. The straight solid line corresponds to $\alpha_{s}^{0}=1.5$, while the straight dashed line corresponds to $\alpha_{s}^{\text {diff }}=0.45$.

vertical scale in Fig. 9 is logarithmic, the sum in Eq. (9) will essentially be dominated by the largest of the two terms, as one can easily see in Fig. 9. Therefore, the large scale behavior of the complete interface is totally dominated by the scaling behavior of the pattern component. On the other hand, the structure factor $S(k, t)$ should crossover to that of the local fluctuations $\langle\hat{y}(k, t) \hat{y}(-k, t)\rangle$ at short wavelengths. The structure factor of the pattern component shifts downwards with time because $\alpha<\alpha_{\mathrm{s}}$. This is not the case for the structure factor of the local fluctuations, which scales according to the Family-Vicsek' ansatz with $\alpha=\alpha_{\text {s }} \approx 0.45$ is fulfilled. Thus the length scale at which the local fluctuations dominate the structure factor should grow with time. Whether the asymptotic scaling behavior of the facets can be observed in the limit $t \rightarrow \infty$ for a finite system depends on the relative strength of the local fluctuations.

\section{LYAPUNOV VECTORS}

So far we have studied the dynamics of random infinitesimal perturbations, which is equivalent to study the dynamics of the most unstable direction in tangent space, i.e., the first LV. We now devote this section to briefly describe the dynamics of further unstable directions that growth at slower rates.

Let us start by introducing a few key quantities that shall be used in our analysis. The evolution equation for an initially random infinitesimal perturbation, Eq. (1), can be written in vectorial form as $\delta \mathbf{u}(t+1)=\mathbf{J}[\mathbf{u}(t)] \delta \mathbf{u}(t)$, where $\mathbf{J}[\mathbf{u}(t)]$ is just the Jacobian evaluated on the trajectory $\mathbf{u}(t)$ at time $t$. Since infinitesimal perturbations evolve linearly there exists a linear operator $\mathbf{M}\left(t_{2}, t_{1}\right)$ such that $\delta \mathbf{u}\left(t_{2}\right)=\mathbf{M}\left(t_{2}, t_{1}\right) \delta \mathbf{u}\left(t_{1}\right)$, given by $\mathbf{M}\left(t_{2}, t_{1}\right)=\prod_{t_{i}=t}^{t_{2}-1} \mathbf{J}\left[\mathbf{u}\left(t_{i}\right)\right]$. Oseledec's theorem [30] implies that the (symmetric) limit operator $\lim _{t_{2} \rightarrow \infty}\left[\mathbf{M}\left(t_{2}, t_{1}\right) \mathbf{M}^{*}\left(t_{2}, t_{1}\right)\right]^{1 / 2\left(t_{2}-t_{1}\right)}$, where $\mathbf{M}^{*}$ denotes the adjoint of $\mathbf{M}$, does exist and the logarithms of its eigenvalues are the Lyapunov exponents $\lambda_{n}(n=1,2, \ldots, L)$ and the eigenvectors are the so-called backward Lyapunov vectors $\mathbf{b}_{n}$ $[4,6]$. In what follows, we will consider standard ordering of the Lyapunov exponents $\lambda_{1} \geqslant \lambda_{2} \geqslant \cdots \geqslant \lambda_{L}$, and the corresponding vectors. We use standard numerical techniques to 


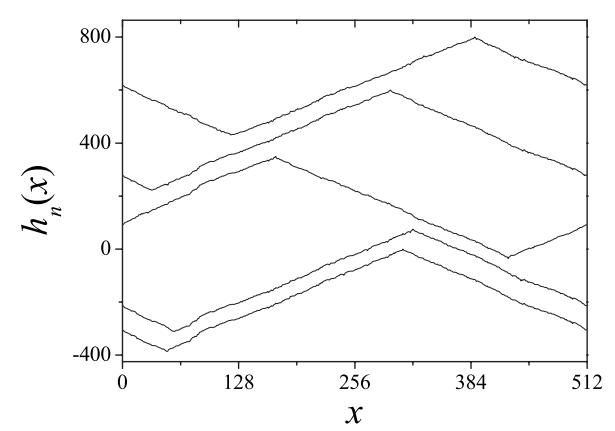

FIG. 10. The surfaces $h_{n}(x)$ corresponding to the first five Lyapunov vectors are plotted (from bottom $n=1$ to top $n=5$ ). Note that all the interfaces converge to a triangular structure. The data presented were obtained for model B and have been shifted for better visibility.

calculate Lyapunov exponents and vectors [31,32].

In general, in order to learn something about the physical properties of a chaotic system, one should study the so called characteristic or covariant LVs, which were first introduced by Ruelle [33] (see also Ref. [4] for a review). Very recent studies have shown that backward LVs might suffer from artifacts, introduced by the orthogonality imposed during the construction of these vectors, which is absent in the case of characteristic LVs $[11,34]$. The latter vectors are, however, very hard to compute in large extended systems. Our preliminary numerical study of the characteristic LVs in small systems indicates that characteristic and backward vectors are identical for each realization of disorder. This might be related to the disorder that breaks the translational invariance existing in homogeneous systems, which leads to strong and static localization of errors at different positions (see details below). Therefore, here we focus on backward LVs in order to be able to access larger system sizes.

As we have discussed in Secs. III and IV, in inhomogeneous systems the main LV, the one corresponding to the most unstable direction, converges to a faceted structure asymptotically dominated by one single facet in the limit $t$ $\rightarrow \infty$. In Fig. 10 we plot the LV surfaces $h_{n}(x, t)$ $=\ln \left[\left|b_{n}(x, t)\right|\right], n \in\{1,2,3,4,5\}$, corresponding to the first five backward LVs for model B at some arbitrary time $t$ during evolution in a system of 512 coupled maps. The surface profiles in this plot are arbitrarily shifted in the vertical direction to aid comparison. In the long time limit the interface $h_{1}(x, t)$, the first LV surface, naturally coincides with the interface $h(x, t)$ corresponding to an arbitrary initially random perturbation. It should be observed that not only the first $\mathrm{LV}$, but each surface $h_{n}(x, t)$ corresponding to the $n$th $\mathrm{LV}$ indeed converges to a structure dominated by a single triangle as well. This is actually the case for all the inhomogeneous models studied in this paper.

Interestingly, the location of the cusp of the triangles, where the LVs spatially localize, is not arbitrary. It is interesting to compare the positions of the global maxima of the interface profiles with the values of the quenched disorder at those sites. For model C, i.e., the RT case, the site where the first LV takes its maximum value coincides with the site $x$ at which the coupling $\epsilon_{x}$ takes its lowest value, the site where the second LV takes its maximum coincides with the site where $\epsilon_{x}$ takes its second lowest value, and so forth. This is easy to interpret: since errors get trapped at sites with lower diffusion, the result is that the LVs strongly localize and utterly get frozen at those sites.

A very similar correlation effect between error localization and disorder can be seen for model B, i.e., the RB case. Note that in the case of RB disorder the diffusion coefficient at a given site $x$ is the sum $\epsilon_{x+1}+\epsilon_{x}$ of the local diffusive couplings connecting $x$ to any of the two neighboring sites. In fact, we find that the site where the first LV takes its maximum coincides with the site $x$ where the diffusivity $\epsilon_{x+1}+\epsilon_{x}$ takes its lowest value, the site where the second LV takes its maximum value coincides with the site where $\epsilon_{x+1}$ $+\epsilon_{x}$ takes its second lowest value and so forth. Thus, the on-site growth velocity is again controlled by the probability for the perturbation to get trapped at that site.

One would expect that a similar association of LVs maxima with disorder extrema should also exist for model A. However, things are not that straightforward in this case. We recall that in model A the disorder is introduced in the map constants and not in the diffusive couplings, which are identical along the system. We think that due to the dominance of small scale fluctuations up to quite large length scales for model A (see Fig. 5) as compared with the situation for models B and C, the maxima get smoothed out and an extended neighborhood, rather than just one single site, controls the final position where the LVs get frozen at long times. In other words, local synchronization of neighboring sites over short scales makes it difficult to simply identify the region where, at a coarse-grained level, the vectors will get asymptotically trapped in the case of disorder of type A.

\section{TORWARD A PHENOMENOLOGICAL STOCHASTIC FIELD THEORY}

The evolution of perturbations in homogeneous SECSs is known to be described in statistical terms by the multiplicative stochastic equation

$$
\frac{\partial}{\partial t} \delta u=\partial_{x x} \delta u+\xi(x, t) \delta u,
$$

where $\xi(x, t)$ is a noise term that accounts for the chaotic fluctuations along the trajectory and is assumed to be $\delta$ correlated in space and time $\left\langle\xi(x, t) \xi\left(x^{\prime}, t^{\prime}\right)\right\rangle=2 \sigma \delta\left(x-x^{\prime}\right) \delta(t$ $\left.-t^{\prime}\right)$. Pikovsky and Kurths [7] and Pikovsky and Politi [8] proposed this equation as the proper candidate for modeling the dynamics of the first Lyapunov vector from a statistical perspective. They showed that it actually reproduces the main statistical properties of SECS in a variety of systems [8]. This equation mimics the linear equations in tangent space for the dynamics of infinitesimal perturbations in spatiotemporal chaotic systems.

An important feature of Eq. (10) is that, under a HopfCole transformation $h=\ln |\delta u|$ it maps into the KPZ equation

$$
\frac{\partial}{\partial t} h=\left(\partial_{x} h\right)^{2}+\partial_{x x} h+\xi(x, t),
$$

which ultimately justifies why the log-transformed (main) Lyapunov vector of many spatiotemporal chaotic systems 
scales in space and time as a KPZ surface [7-11]. Interestingly, Eq. (10) also appears in the context of the classical problem of the directed polymer in a random potential [35] (also see Ref. [36] for a detailed review).

We now explore extensions of this type of Langevin equations to the case of disordered systems. A phenomenological stochastic field theory of this kind should be helpful in order to identify the essential symmetries and mechanisms that determine the dynamics of perturbations in chaotic disordered systems. The existence of scale invariance strongly encourages this approach. Therefore, microscopic details of the models are expected to be irrelevant at a sufficiently coarsegrained scale, as occurs in the case of homogeneous systems.

In the case of model A, Eq. (1) can be considered as a straightforward discrete version of

$$
\frac{\partial}{\partial t} \delta u=\partial_{x x}\left[\partial_{u} f(x, u) \delta u\right]+\left[\partial_{u} f(x, u)-1\right] \delta u .
$$

It is important to note that the noisy term $\partial_{u} f(x, u)$ $\left.\equiv \partial_{y} f(x, y)\right|_{u(x, t)}$ depends implicitly on time through the trajectory $u(x, t)$ and so, it includes both an annealed and a quenched component. The origin of the latter is the inhomogeneous character of the coupled elements: Sites are more or less chaotic depending on their position $x$ in the system. For instance, in the particular case of the logistic map we have $\partial_{u} f(x, u)=c(x)[1-2 c(x) u]$, where $c(x)$ is the local map parameter. We argue that in the hydrodynamic limit the quenched part of the noise dominates over the annealed one. The reason being that the quenched character of the disorder generates long-range temporal correlations at each site, which are expected to control the dynamics over the shortrange correlations of the annealed random terms.

These arguments suggest that for type A models the long time limit of main LV should be generically described by a multiplicative Langevin equation such as

$$
\frac{\partial}{\partial t} \delta u=\partial_{x x}[\zeta(x) \delta u]+\partial_{x x} \delta u+\zeta(x) \delta u,
$$

where $\zeta(x)$ represents the quenched disorder. This equation can be further simplified since the disordered diffusion term $\partial_{x x}[\zeta(x) \delta u]$ is irrelevant as compared with $\partial_{x x} \delta u$. To be precise, in the case of weak disorder that we study here the inhomogeneous diffusion term simply renormalizes to an effective constant diffusion term [47]. Finally we arrive at our stochastic field theory proposal for model A type of systems

$$
\partial_{t} \delta u=D \partial_{x x} \delta u+\zeta(x) \delta u,
$$

where $D$ is an effective diffusion constant and $\zeta(x)$ is a quenched columnar disorder with $\delta$ correlations.

Interestingly, Eq. (13) appears in the context of diffusion in random trapping or amplifying quenched potentials [37-44]. After the Hopf-Cole transform of the perturbation field, $\delta u=\exp (\mu h / D)$, one obtains

$$
\partial_{t} h(x, t)=\partial_{x x} h+\mu\left(\partial_{x} h\right)^{2}+\zeta(x),
$$

which is a close relative of the KPZ equation, but here the additive noise term $\zeta(x)$ is quenched and delta correlated, $\left\langle\zeta(x) \zeta\left(x^{\prime}\right)\right\rangle=2 \theta \delta\left(x-x^{\prime}\right)$. This equation has attracted some in- terest in the past, as it also describes the free energy of directed and undirected polymers of length $t$ in the presence of columnar disorder $[22,29,45,46]$.

Only very recently [29], it has been realized that the surface described by Eq. (14) exhibits anomalous scaling exponents induced by the self-organization in a faceted structure. In Ref. [29] we studied Eq. (14) by means of extensive simulations and determined the critical exponents to be $z$ $=1.35 \pm 0.05, \quad \alpha=1.05 \pm 0.05$, and $\alpha_{s}=1.50 \pm 0.05$. These values are in excellent agreement with the values reported here for the critical exponents of the main LV surface in SECSs of type A (see Fig. 5). This strongly supports our claim that, in fact, Eq. (13) is a minimal model that captures the essential ingredients dominating the evolution of errors in inhomogeneous models of type A [48].

As it has already been discussed in Ref. [29], the introduction of an additional annealed noise term in Eq. (14) is irrelevant in the renormalization group sense, but leads to an increase of the typical size over which the local random fluctuations (discussed in Sec. V) dominate. Hence, for a finite size system the asymptotic scaling behavior may not be observable if the thermal noise amplitude is too large. This is of some importance for the situations we are interested in because, when considering the evolution of perturbations in SECS, there will always be some temporally fluctuating noise as explained above. Thus, even if quenched disorder is present in the studied system, it may be very weak in comparison with the temporally fluctuating one. In that case the behavior described here would only be observable in very large systems.

Moreover, it should be noticed that, although the roughness exponents $\alpha, \alpha_{\text {loc }}$, and $\alpha_{s}$ seem to be the same within error bars for the three models of disorder studied in this paper, the dynamic exponent $z$ does take different values. This suggests that the disorder symmetries that distinguish models A, B, and C are indeed relevant and lead to different nonequilibrium universality classes.

One can try to formulate a stochastic-field evolution equation similar to Eq. (13) for models B and C, but where the disorder enters in a different form into the diffusion and the multiplicative term in order to reflect the different symmetries of the coupling in these two models. Unfortunately, we have not been able to find the correct equations reproducing the scaling behavior of the LV surfaces for disordered systems in the class B and C. Similar arguments might be used to write down the stochastic equation

$$
\partial_{t} \delta u=\partial_{x}\left[\zeta(x) \partial_{x} \delta u\right]+\xi(x, t) \delta u
$$

for model B and

$$
\partial_{t} \delta u=\partial_{x x}[\zeta(x) \delta u]+\xi(x, t) \delta u
$$

for model $\mathrm{C}$, where $\xi(x, t)$ is a noise term. We have investigated the scaling properties of Eqs. (15) and (16) by means of numerical simulations and found that, while these simple stochastic models generically lead to faceted surfaces with the expected roughness exponents, they are unable to reproduce the correct dynamic exponent of disordered SECS in the class B nor $\mathrm{C}$, but instead yield the same dynamical exponent as Eq. (13). We believe that the reason behind this 
disagreement lies in the existence of correlations between the quenched and annealed fluctuating terms that are not incorporated in this naive approach. These nontrivial correlations might also explain the, at least at first sight, counter intuitive result that different dynamic exponents are observed in SECS of class B and C. Some remarks regarding this point are now in order.

Classical diffusion in random media involves the study of equations such as (15) and (16), but where the multiplicative term $\xi(x, t) \delta u$ is absent. It is well known [21] that in the long time limit, dynamics of RB and RT models is identical, even for strong disorder. Despite the different symmetries involved, the large scale physics is the same. However, our numerical results indicate that model B (RB symmetry) and model C (RT symmetry) in SECS seemingly belong to different universality classes. We claim that the multiplicative noise, which is generically coupled to the quenched disorder in the systems we have studied, breaks the duality between $\mathrm{RB}$ and RT type of disorder in this case, leading to distinct dynamical exponents for the two different diffusion configurations.

\section{CONCLUSIONS}

We have studied the evolution of infinitesimal perturbations in inhomogeneous spatially extended systems exhibiting space-time chaos. Inhomogeneity is introduced by means of a quenched disorder. We have considered one-dimensional coupled-map lattices as a simple and computationally convenient model system to analyze some aspects of chaos in the presence of disorder. In this regard, three different classes of models have been investigated by means of extensive numerical simulations. In all cases we find a strong localization behavior characterized by an exponential spatial profile around some localization centers. In the long time limit the perturbation concentrates around one single final attracting center. We have also studied the second, third, and so on backward LVs corresponding to most rapidly expanding directions in tangent space and found a correspondence between the localization centers and the positions in space cor- responding to increasing minima of the diffusion in the particular disorder realization.

Note that the strong localization behavior described here is essentially different from the dynamic localization observed in the case of homogeneous SECS. In the latter, perturbations do also localize on just a few sites, but the position of these sites keeps fluctuating in time. However, in the presence of quenched disorder the sites where perturbations localize are fixed by the corresponding realization of the disorder.

Moreover, by a standard mapping (Hopf-Cole transform) of the perturbation into a growing surface we found that the LV associated surfaces self-organize in a faceted structure, at variance with what occurs in homogeneous (nondisordered) systems where one generically obtains a surface in the universality class of KPZ. Interestingly, this faceted surface was found to exhibit coarsening and anomalous kinetic roughening in agreement with previous theoretical predictions [28] for this type of scale-invariant structure.

The evolution of infinitesimal perturbations in spatially extended chaotic systems with quenched disorder may be described at a coarse-grained level as a diffusion process in a random potential. Note, however, that the value of the dynamical exponent $z$ depends on how disorder is introduced. The reason for this is possibly the influence of the disorder distribution on the temporal dependence of the coarsening, as described in Ref. [22] in the context of diffusion (see also Ref. [29] for a discussion relevant for the present work)

We think that strong localization and anomalous scaling should play a role, e.g., in realistic weather models, where quenched disorder is included in the form of inhomogeneous boundary conditions representing certain geographical and topographical conditions.

\section{ACKNOWLEDGMENTS}

We thank D. Pazó for enlightening discussions and M. A. Muñoz for his support and comments on this work. Financial support from the Ministerio de Educación y Ciencia (Spain) under project Nos. FIS2006-12253-C06-04 and CGL200764387/CLI is acknowledged.
[1] T. Bohr, M. H. Jensen, G. Paladin, and A. Vulpiani, Dynamical Systems Approach to Turbulence (Cambridge, Cambridge, 1988).

[2] U. Frish, Turbulence (Cambridge University Press, Cambridge, 1995).

[3] E. Kalnay, Atmospheric Modeling, Data Assimilation and Predictability (Cambridge University Press, Cambridge, 2003).

[4] J.-P. Eckmann and D. Ruelle, Rev. Mod. Phys. 57, 617 (1985).

[5] E. Ott, Chaos in Dynamical Systems (Cambridge University Press, Cambridge, 1993).

[6] B. Legras and R. Vautard, in Predictability, ECWF Seminar, edited by T. Palmer (ECMWF, Reading, UK, 1996), Vol. I, pp. $135-146$

[7] A. S. Pikovsky and J. Kurths, Phys. Rev. E 49, 898 (1994).
[8] A. Pikovsky and A. Politi, Nonlinearity 11, 1049 (1998).

[9] A. Pikovsky and A. Politi, Phys. Rev. E 63, 036207 (2001).

[10] A. D. Sánchez, J. M. López, M. A. Rodríguez, and M. A. Matías, Phys. Rev. Lett. 92, 204101 (2004).

[11] I. G. Szendro, D. Pazó, M. A. Rodríguez, and J. M. López, Phys. Rev. E 76, 025202(R) (2007).

[12] M. Kardar, G. Parisi, and Y.-C. Zhang, Phys. Rev. Lett. 56, 889 (1986).

[13] J. G. Charney, in Dynamic Meteorology, edited by P. Morel (Reidel, Boston, 1973), pp. 97-351.

[14] J. Pedlosky, Geophysical Fluid Dynamics (Springer-Verlag, Berlin, 1979).

[15] B. Legras and M. Ghil, J. Atmos. Sci. 42, 433 (1985).

[16] Y. Brainman, J. F. Linder, and W. L. Ditto, Nature (London) 
378, 465 (1995).

[17] K. Wiesenfeld, P. Colet, and S. H. Strogatz, Phys. Rev. Lett. 76, 404 (1996).

[18] N. Mousseau, Phys. Rev. Lett. 77, 968 (1996).

[19] V. Ahlers, R. Zillmer, and A. Pikovsky, Phys. Rev. E 63, 036213 (2001).

[20] J. W. Haus and K. W. Kehr, Phys. Rep. 150, 263 (1987).

[21] J.-P. Bouchaud and A. Georges, Phys. Rep. 195, 127 (1990).

[22] J. Krug and T. Halpin-Healy, J. Phys. I 3, 2179 (1993).

[23] F. Family and T. Vicsek, J. Phys. A 18, L75 (1985).

[24] A.-L. Barabási and H. E. Stanley, Fractal Concepts in Surface Growth (Cambridge University Press, Cambridge, 1995).

[25] J. G. Amar, P.-M. Lam, and F. Family, Phys. Rev. E 47, 3242 (1993).

[26] M. Schroeder et al., Europhys. Lett. 24, 563 (1993).

[27] S. Das Sarma, S. V. Ghaisas, and J. M. Kim, Phys. Rev. E 49, 122 (1994).

[28] J. J. Ramasco, J. M. López, and M. A. Rodríguez, Phys. Rev. Lett. 84, 2199 (2000).

[29] I. G. Szendro, J. M. López, and M. A. Rodríguez, Phys. Rev. E 76, 011603 (2007).

[30] V. Oseledec, Trans. Mosc. Math. Soc. 19, 179 (1968).

[31] G. Benettin, L. Galgani, A. Giorgilli, and J.-M. Strelcyn, Meccanica 15, 9 (1980).

[32] K. Geist, U. Parlitz, and W. Lauterborn, Prog. Theor. Phys. 83, 875 (1990).

[33] D. Ruelle, Publ. Math., Inst. Hautes Etud. Sci. 50, 275 (1979).

[34] F. Ginelli, P. Poggi, A. Turchi, H. Chate, R. Livi, and A. Politi, Phys. Rev. Lett. 99, 130601 (2007).

[35] M. Kardar and Y. C. Zhang, Phys. Rev. Lett. 58, 2087 (1987).
[36] T. Halpin-Healy and Y. C. Zhang, Phys. Rep. 254, 215 (1995)

[37] Y. C. Zhang, Phys. Rev. Lett. 56, 2113 (1986).

[38] W. Ebeling, A. Engel, B. Esser, and R. Feistel, J. Stat. Phys. 37, 369 (1984).

[39] A. Engel and W. Ebeling, Phys. Rev. Lett. 59, 1979 (1987).

[40] R. Tao, Phys. Rev. Lett. 61, 2405 (1988).

[41] R. Tao, Phys. Rev. Lett. 63, 2695 (1989).

[42] R. Tao, Phys. Rev. A 43, 5284 (1991).

[43] M. N. Rosenbluth, Phys. Rev. Lett. 63, 467 (1989).

[44] A. Valle, M. A. Rodríguez, and L. Pesquera, Phys. Rev. A 43, 2070 (1991).

[45] M. E. Cates and R. C. Ball, J. Phys. (France) 49, 2009 (1988).

[46] T. Nattermann and W. Renz, Phys. Rev. A 40, 4675 (1989).

[47] On the contrary, in the case of strong disorder the existence of strongly trapping sites gives a subdiffusive contribution to the dynamic exponent [20,21]. Again, this term would be irrelevant in the long time limit, which is dominated by the constant diffusion term in Eq. (12).

[48] It is worth to mention here that there are analytical results [22] concerning Eq. (14) showing that the correlation length scales as $\sim t /(\ln t)^{3 / 4}$ for a Gaussian disorder, while slightly different logarithmic corrections are obtained for different disorder distributions. This implies that $z \approx 1.35$ has to be seen as an effective exponent, and as such will not be universal. Nonetheless, these corrections are very weak and extremely difficult to distinguish from purely power-law growth, even with several decades of scaling. We kindly direct the interested reader to Ref. [29] for further details. 